\title{
REVIEWS
}

\section{Migration, maintenance and recall of memory T cells in peripheral tissues}

\section{David L. Woodland and Jacob E. Kohlmeier}

\section{Abstract | After the resolution of an immune response, antigen-specific memory $T$ cells} persist at many sites in the body. The antigen-specific memory T-cell pool includes memory T cells that preferentially reside in peripheral tissues, such as the skin, gut and lungs, where they provide a first line of defence against secondary pathogen infection. Determining how peripheral memory $T$ cells are regulated is essential for our understanding of host-pathogen interactions and for vaccine development. In this Review, we discuss recent insights into the generation, control and recall of peripheral T-cell memory responses.
Lamina propria

A layer of connective tissue between the intestinal epithelium and the intestinal muscularis mucosae layer that contains various myeloid and lymphoid cells, including macrophages, dendritic cells, $T$ cells and $B$ cells.

Parabiosis experiments Studies of parabiotic mice which are surgically joined and therefore have a common blood circulation.
Trudeau Institute, 154 Algonquin Avenue, Saranac Lake, New York 12983, USA. Correspondence to D.L.W. and J.E.K.

e-mails: dwoodland@ trudeauinstitute.org; jkohlmeier@trudeauinstitute. org

doi: $10.1038 / n$ ri2496
The mucosal surfaces of the respiratory, intestinal and genital tracts, as well as abrasions of the skin, are major portals of entry into the body for many pathogens. Given that the total surface area of all of these peripheral sites can be more than $400 \mathrm{~m}^{2}$, effective surveillance poses a formidable challenge for the immune system. During an infection, the immune system not only makes a rapid pathogen-specific response at these sites to destroy the invading pathogen, but also generates long-lived memory $\mathrm{T}$ cells that can persist at peripheral sites for many months. However, our understanding of how these peripheral memory $\mathrm{T}$ cells are regulated is still in its infancy.

The development and propagation of an adaptive immune response specific for an invading pathogen is a highly orchestrated process that involves the activation and proliferation of $\mathrm{T}$ cells and their subsequent migration to sites of inflammation. The migration of $\mathrm{T}$ cells to peripheral sites is regulated by many factors, including adhesion molecules and chemokine receptors that are expressed by T cells, and integrins, selectins and chemokines that are expressed by cells at various peripheral sites. Effector T cells are preferentially attracted to sites of inflammation and they are highly promiscuous in their migratory patterns. For example, effector T cells can enter all inflamed non-lymphoid tissues, regardless of where the T cells initially encountered antigen ${ }^{1}$. This allows the immune response to remain flexible should the infection spread to other tissues. After pathogen clearance, the recruitment of effector T cells to the site of infection is slowed by both decreasing levels of inflammation and the sequestration of excess chemokines by decoy receptors and apoptotic cells ${ }^{2,3}$. Antigen-specific T cells that remain in the body after the contraction of the effector T-cell population are then maintained indefinitely as long-lived memory T cells, which can generate a rapid recall response to secondary pathogen challenge ${ }^{4-6}$. However, in contrast to effector $\mathrm{T}$ cells, the migration of memory $\mathrm{T}$ cells to non-lymphoid tissues is more restricted, raising questions as to how the trafficking of memory $\mathrm{T}$ cells to peripheral sites is controlled and what role peripheral memory $\mathrm{T}$ cells have in the protection against secondary pathogen challenge.

In this Review, we discuss recent evidence that describes the cellular interactions that are required for the generation of peripheral memory T-cell populations, the mechanisms by which these cells are maintained, the role of these cells in resistance to secondary infections and how these insights can be used to improve existing vaccination strategies.

\section{Memory T-cell migration to peripheral sites}

Although it has been known for a long time that memory $\mathrm{T}$ cells are widely disseminated throughout the body, studies in several model systems have shown that the site of infection and the type of tissue that is infected by the pathogen greatly influence the distribution of the resulting pathogen-specific memory T-cell population. For example, infection of the lungs, intestines or skin results in the establishment of markedly greater numbers of antigen-specific memory $\mathrm{T}$ cells at the site of infection (that is, the lung airways, lamina propria or dermis, respectively) than at other peripheral sites that did not contain replicating pathogens ${ }^{7-9}$. By contrast, the numbers of memory $\mathrm{T}$ cells established in organs such as the spleen, liver and bone marrow by these different peripheral infections are often quite similar. Furthermore, memory T-cell transfer and parabiosis experiments have shown that the migration of circulating memory $\mathrm{T}$ cells from the circulation to some peripheral 
sites, such as the intestines, skin and lung airways, is limited ${ }^{10-12}$ (FIG. 1). Together, these studies show that the controlled entry of memory T cells is a feature shared by several peripheral tissues. Therefore, although memory $\mathrm{T}$ cells generated by different infections have a common ability to migrate to many different tissues, there are specific tissues to which the entry of circulating memory $\mathrm{T}$ cells is highly restricted.

These differential migratory patterns indicate that cell-surface molecules must regulate the entry of T cells into peripheral sites. Indeed, many studies have investigated the relationship between antigen-specific T-cell populations in lymphoid and peripheral tissues and the expression of adhesion molecules and chemokine receptors that control the localization of these T cells. Some of these studies have identified molecules that seem to have a general role in the trafficking of memory $\mathrm{T}$ cells from the circulation to the surrounding tissue. For example, the expression levels of CD 44 , aL 32 -integrin

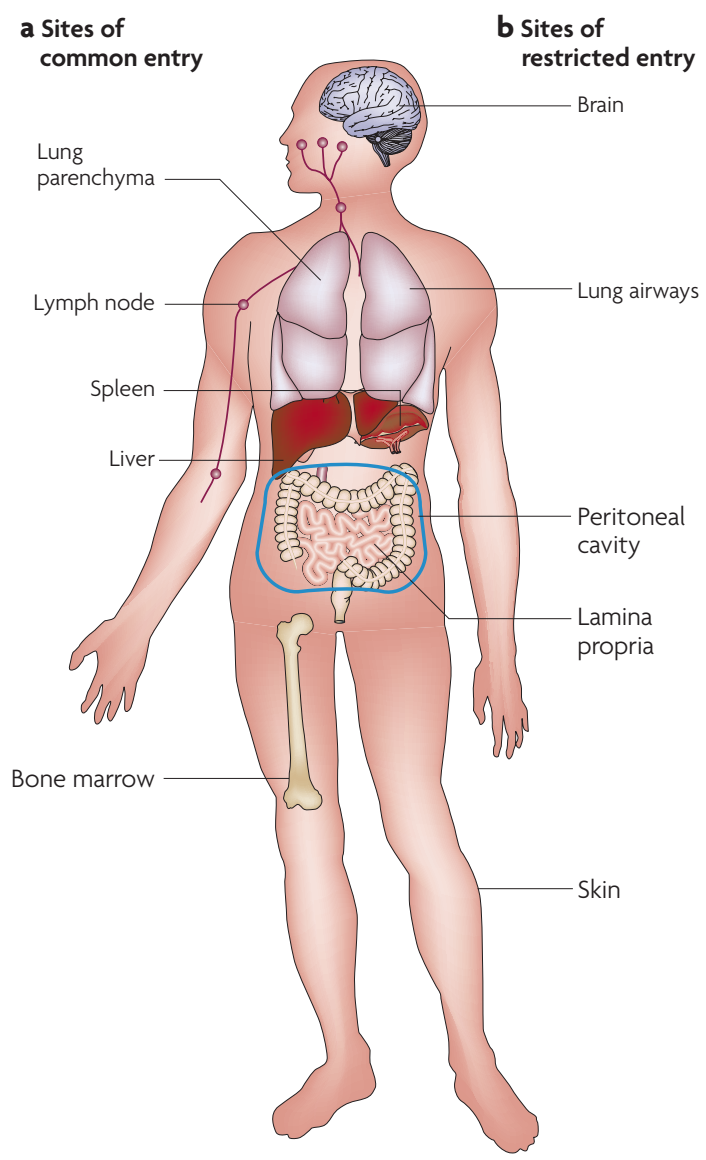

Figure 1 | Sites of common and restricted entry for circulating memory T cells. The ability of memory T cells to migrate from the circulation into the surrounding tissue varies depending on the tissue. a | For certain sites, migration into the tissue is strictly dependent on general molecules expressed by memory $T$ cells, regardless of the route of infection or the tissue tropism of the pathogen. b| By contrast, other peripheral sites require that memory T cells express tissue-specific adhesion molecules and/or chemokine receptors for optimal migration from the circulation into the peripheral tissue. (also known as LFA1), $\alpha 4 \beta 1$-integrin (also known as VLA4) and P-selectin glycoprotein ligand 1 (PSGL1) are increased on antigen-experienced $\mathrm{T}$ cells $\mathrm{s}^{13-17}$, and the broad pattern of expression of these molecules indicates that they are not selective markers for migration into individual tissues. By contrast, several seminal studies discussed below have shown that the expression of specific combinations of adhesion molecules and chemokine receptors is associated with $\mathrm{T}$-cell migration to distinct peripheral sites.

Imprinting to the skin and gut. The expression of different combinations of adhesion molecules and chemokine receptors can mediate the control of T-cell trafficking (TABLE 1), and it has been well demonstrated that $\mathrm{T}$ cells from the skin and gut have distinctive patterns of expression of these molecules ${ }^{18}$. Furthermore, the posttranslational modification (such as glycosylation) of these surface proteins can markedly alter their interactions with specific ligands ${ }^{19}$. As the molecular mechanisms that drive these expression patterns, which are often known as 'imprinting', have been expertly reviewed elsewhere ${ }^{20,21}$, we do not describe these processes in detail. However, an understanding of the surface receptors that are involved in T-cell trafficking to these sites, and of the different cell types that influence their expression, is relevant to our discussion. T cells programmed to migrate selectively to the skin express P- and E-selectin ligands that have undergone specific protein modifications ${ }^{22}$ that allow them to bind to E-selectin expressed by vascular endothelial cells ${ }^{23}$; these modified ligands are known as cutaneous lymphocyte-associated antigen (CLA). Skin-homing T cells also express CC-chemokine receptor 4 ( $\underline{\text { CCR } 4})$ and/or CCR10, which interact with the dermal-associated chemokines CC-chemokine ligand 17 (CCL17) and CCL27, respectively ${ }^{24,25}$. By contrast, guthoming $\mathrm{T}$ cells express $\underline{\alpha} 4 \beta 7$-integrin, which binds to mucosal vascular addressin cell adhesion molecule 1 (MADCAM1 $)^{26,27}$, and the chemokine receptor CCR9, which interacts with the gut-associated chemokine CCL25 (REFS 28,29). Interestingly, the mechanisms that lead to the acquisition of a skin- or gut-homing phenotype by memory $\mathrm{T}$ cells can also suppress the expression of receptors that are associated with the opposing phenotype $\mathrm{p}^{30}$. Furthermore, the tissue tropism of established memory T cells is not fixed and can be modified during a secondary pathogen challenge, depending on the route of infection and the anatomical location in which pathogen-specific $\mathrm{T}$ cells interact with antigenbearing dendritic cells (DCs) ${ }^{31,32}$. So, the plasticity of tissue-tropic phenotypes allows memory $\mathrm{T}$ cells to be reprogrammed to respond efficiently to secondary infections that are localized at different anatomical sites from the primary infection.

The local microenvironment in which $\mathrm{T}$ cells are primed is an important factor for determining the development of tissue-specific migratory capabilities. For example, pulmonary infection with rotavirus, which is normally an intestinal pathogen, generates antigenspecific $\mathrm{T}$ cells with a different trafficking profile to the memory $\mathrm{T}$ cells that are generated after an intestinal 
Table 1 | Receptors involved in the migration of peripheral memory $\mathbf{T}$ cells

\begin{tabular}{|c|c|c|}
\hline Cell-surface molecule & Receptor & Tissue tropism \\
\hline CD44 & Hyaluronate and fibrinogen & General \\
\hline CD43 & E-selectin & General \\
\hline aL $\beta 2$-integrin (LFA1) & ICAM1 & General \\
\hline P-selectin and E-selectin ligands & P-selectin and E-selectin & General \\
\hline$\alpha 4 \beta 1$-integrin (VLA4) & VCAM1 and fibronectin & General \\
\hline$\alpha 4 \beta 7$-integrin & MADCAM1 & Gut \\
\hline CLA-modified ligands & E-selectin & Skin \\
\hline a1ß1-integrin (VLA1) & Collagen and laminin & Lung* \\
\hline CD62L & PNAd & Lymph node \\
\hline CCR4 & CCL17 & Skin \\
\hline CCR10 & CCL27 and CCL28 & Skin \\
\hline CCR9 & CCL25 & Small bowel \\
\hline CCR7 & CCL19 and CCL21 & Lymph node \\
\hline
\end{tabular}

*It is currently unclear whether this molecule is involved in memory T-cell recruitment to, or survival in, the lung airways. CCL, CC-chemokine ligand; CCR, CC-chemokine receptor; CLA, cutaneous lymphocyte-associated antigen; ICAM1, intercellular adhesion molecule 1; LFA1, lymphocyte function-associated antigen 1; MADCAM1, mucosal vascular addressin cell adhesion molecule 1; PNAd, peripheral lymph node addressin; VCAM1, vascular cell adhesion molecule 1; VLA, very late activation protein.

Homeostatic proliferation The periodic division of cells in the periphery that allows for the long-term maintenance of memory T-cell populations. This process is mediated by the cytokines IL-7 and IL-15. infection ${ }^{33}$. So, the route of infection and site of replication, rather than the pathogen itself, are the main factors that influence the development of tissue tropism of the memory T-cell response.

Most of the aforementioned studies that investigated the migration of $\mathrm{T}$ cells to the skin and gut have shown that DCs isolated from the lymph nodes that drain these tissues are sufficient to programme T cells with a tissue-tropic phenotype ${ }^{34,35}$. However, several recent reports have shown that non-haematopoietic peripheral tissue cells and lymph node-resident stromal cells can also influence the tissue tropism of $\mathrm{T}$ cells ${ }^{36,37}$. For example, one elegant study recently showed that peripheral lymph nodes transplanted into the gut mesenteries could not generate $\mathrm{T}$ cells with a gut-homing phenotype, despite the presence of gut-derived DCs in the transplanted lymph nodes ${ }^{38}$. These studies indicate that the programming of antigen-presenting cells such as DCs by non-haematopoietic cells in the local environment is a prerequisite for imparting tissue tropism to antigenspecific T cells.

Imprinting to other peripheral sites. The identification of tissue-homing T-cell phenotypes for the skin and gut has led to considerable interest regarding whether $\mathrm{T}$ cells can be imprinted to migrate selectively to other peripheral sites. Although the mechanisms involved have not yet been identified, there is evidence to suggest that T-cell imprinting does occur for other sites of restricted entry, such as the lung airways and central nervous system. For example, T cells that were activated in the cervical lymph nodes acquired preferential memory $\mathrm{CD}^{+} \mathrm{T}$ cells were found to be enriched in the cerebrospinal fluid of humans with no known tropism for the central nervous system ${ }^{39}$. In addition, neurological disorders ${ }^{40}$, and large numbers of memory $\mathrm{CD}^{+} \mathrm{T}$ cells are maintained in the brains of mice for several months after clearance of dengue virus infection $^{41}$. A comparison of lymphocytes from the lung airways and from the peripheral blood of humans has shown that memory $\mathrm{CD} 8^{+} \mathrm{T}$ cells specific for respiratory pathogens, but not memory $\mathrm{CD} 8^{+} \mathrm{T}$ cells specific for systemic pathogens, are selectively enriched in the lung airways ${ }^{42}$. One adhesion molecule that could direct the recruitment of memory $\mathrm{T}$ cells to the lungs is a1 $\beta 1$-integrin (also known as VLA1), which is highly expressed by respiratory virus-specific memory $\mathrm{CD}^{+}$ $\mathrm{T}$ cells in the airways ${ }^{43}$. In addition, blocking VLA1 interactions with VLA1-specific antibodies inhibited the induction of protective immunity during a secondary pathogen challenge. However, it is currently unclear whether VLA1 is required for the migration of memory $\mathrm{T}$ cells to the airways or whether its main role is in the retention of $\mathrm{T}$ cells after migration to the site.

\section{Maintenance of peripheral T-cell memory}

Although pathogen-specific memory T cells are relatively quiescent, they nonetheless undergo many changes in phenotype, function and location over time. Once established, the overall size of the memory T-cell pool that is specific for a particular antigen remains relatively constant and it is maintained through homeostatic proliferation driven by interleukin-7 (IL-7) and IL-15 (REF. 44). However, dividing the total antigen-specific memory T-cell pool into peripheral and lymphoid tissueresident populations reveals a much more dynamic picture. Although memory T cells in peripheral and lymphoid sites seem to undergo a similar rate of homeostatic turnover ${ }^{45,46}$, the number of antigen-specific $\mathrm{T}$ cells at peripheral sites steadily decreases in the months after pathogen clearance. As this waning of peripheral T-cell memory correlates with decreased protection from secondary infections (discussed below), it is essential to understand the mechanisms that regulate the distribution and survival of pathogen-specific memory $\mathrm{T}$ cells in the periphery.

Memory T cells localized in peripheral tissues, particularly at sites such as the mucosal epithelium that interface directly with the surrounding environment, are often exposed to more strenuous physiological conditions than are memory T cells in lymphoid organs. For example, memory $\mathrm{T}$ cells in the lung airways exist at near atmospheric oxygen concentrations and are bathed in hydrophilic surfactant proteins that help to maintain low surface tension in the airway environment. The main pulmonary surfactants, surfactant protein A1 (SP-A1; also known as SFTPA1) and SP-D (also known as SFTPD), also have immunoregulatory functions, which might contribute to cellular stress for T cells in the lung airways ${ }^{47}$. In addition, memory $\mathrm{T}$ cells in the mucosa decrease their expression of the receptors for IL-7 and IL-15, which might prohibit the homeostatic turnover of these $\mathrm{T}$ cells ${ }^{48}$. Evidence to indicate how peripheral memory $\mathrm{T}$ cells survive these pressures was provided by a recent study showing that lymphoid and peripheral memory $\mathrm{T}$ cells have marked differences in their ability to 


\section{Apoptosis}

A common form of cell death, which is also known as intrinsic or programmed cell death. Apoptosis involves cell shrinkage, chromatin condensation in the periphery of the nucleus, cell-membrane blebbing and DNA

fragmentation into multiples of 180 base pairs. Eventually, the cell breaks up into many membrane-bound apoptotic bodies, which are phagocytosed by neighbouring cells.

\section{Annexin V}

A molecule that binds phosphatidylserine, which is usually located on the inner leaflet of the plasma membrane but flips to the outer leaflet during apoptosis. Positive staining with annexin $V$ is an indicator of apoptosis.

Central memory $\mathrm{T}\left(\mathrm{T}_{\mathrm{CM}}\right)$ cells Antigen-experienced CD8+ $T$ cells that lack immediate effector function but can mediate rapid recall responses They also rapidly develop the phenotype and function of effector memory T cells after restimulation with antigen. $\mathrm{T}_{\mathrm{Cu}}$ cells retain the migratory properties of naive cells and therefore circulate through the secondary lymphoid organs

Effector memory $\mathrm{T}\left(\mathrm{T}_{\mathrm{EM}}\right)$ cells

Terminally differentiated T cells that lack lymph-node-homing receptors but express receptors that enable these cells to home to inflamed tissues. $T_{E M}$ cells can exert immediate effector functions without the need for further differentiation. resist apoptosis ${ }^{49}$. T cells in the periphery had decreased levels of annexin $V$ staining and were more resistant to apoptosis. Interestingly, splenic memory $\mathrm{T}$ cells placed in a peripheral environment rapidly acquired increased resistance to apoptosis. Therefore, the ability of memory $\mathrm{T}$ cells to survive in the periphery is regulated after their entry to peripheral sites ${ }^{50}$.

Effector memory and central memory T cells. Memory T cells established after infection can be divided into two subsets based on expression of the lymphoid homing receptors $\underline{\mathrm{CD}} 62 \mathrm{~L}$ and $\mathrm{CCR} 7$ : central memory $\mathrm{T}\left(\mathrm{T}_{\mathrm{CM}}\right)$ cells are $\mathrm{CD}_{62} \mathrm{~L}^{+} \mathrm{CCR} 7^{+}$and preferentially localize to lymphoid tissues, whereas effector memory $T\left(T_{E M}\right)$ cells are $\mathrm{CD}^{2} \mathrm{~L}^{-} \mathrm{CCR}^{-}$and preferentially localize to peripheral tissues $^{51,52}$ (BOX 1). Immediately after pathogen clearance, the antigen-specific memory T-cell pool is comprised mainly of $\mathrm{T}_{\mathrm{EM}}$ cells, which gradually convert to a $\mathrm{T}_{\mathrm{CM}}$ cell phenotype over time. Although the mechanism is still controversial, it seems that this conversion of the systemic memory T-cell pool occurs mostly through the gradual outgrowth of the $\mathrm{T}_{\mathrm{CM}}$-cell population as a result of homeostatic turnover ${ }^{53}$. This phenomenon can have a marked effect on the propensity of memory $\mathrm{T}$ cells to localize to peripheral tissues because, although both $\mathrm{T}_{\mathrm{CM}}$ and $\mathrm{T}_{\mathrm{FM}}$ cells are found in the circulation, the homing receptors expressed by $\mathrm{T}_{\mathrm{CM}}$ cells dictate their preferential localization to lymphoid, rather than peripheral, sites. So, the number of circulating pathogen-specific memory $\mathrm{T}$ cells that selectively traffic to peripheral tissues (that is, $\mathrm{T}_{\mathrm{FM}}$ cells) gradually decreases over time. It should be noted, however, that a recent study showed that repeated vaccination to boost a pre-existing memory T-cell population resulted in the accumulation of large numbers of antigen-specific $\mathrm{T}_{\mathrm{EM}}$ cells $^{54}$. Therefore, re-exposure to a pathogen could generate more $\mathrm{T}$ cells that can localize to peripheral tissues.
Although the gradual loss of systemic $\mathrm{T}_{\mathrm{FM}}$ cells correlates with the finding that the number of peripheral memory $\mathrm{T}$ cells decreases during the first 6 months after infection, animal models have shown that pathogenspecific memory $\mathrm{T}$ cells can be found in small but detectable numbers at some sites, such as the lung airways and intestinal epithelium, long after the systemic population has converted to a $\mathrm{T}_{\mathrm{CM}}$-cell phenotype $\mathrm{e}^{55,56}$. Although it is not clear how peripheral memory $\mathrm{T}$ cells are maintained over time in the skin and gut, this finding is at odds with the observation that memory $\mathrm{T}$ cells in the lung airways have a limited lifespan such that the population must be maintained by continual recruitment from the circulation ${ }^{57}$. It could be that a low frequency of $\mathrm{T}_{\mathrm{EM}}$ cells in the circulation is required to maintain memory $\mathrm{T}$ cells in the lung airways at later times after infection, but it is also possible that $\mathrm{T}_{\mathrm{CM}}$ cells, having shed $\mathrm{CD} 62 \mathrm{~L}$ from their surface and thereby having gained the phenotypic characteristics of $\mathrm{T}_{\mathrm{EM}}$ cells, contribute to memory T-cell populations at peripheral sites. This hypothesis is supported by studies of transgenic mice expressing CD62L in which no differences in the number of peripheral memory $\mathrm{T}$ cells were observed, despite constitutive CD62L expression ${ }^{58}$. Importantly, the migration of systemic memory $\mathrm{T}$ cells to non-lymphoid sites also results in the modulation of their functional properties, in addition to their phenotype ${ }^{59}$ (BOX 1). Therefore, circulating $\mathrm{T}_{\mathrm{CM}}$ cells probably contribute to the maintenance of peripheral memory T-cell populations to a small extent, and once in peripheral tissues, these T cells can mount localized responses to a secondary pathogen challenge.

The role of residual antigen. During chronic infections, persistent antigen stimulation has been shown to be an important factor in shaping the T-cell response. By contrast, during an acute infection, antigen was thought to be cleared from the host quite rapidly after

\section{Box 1 | Functional differences between lymphoid $\mathbf{T}_{\mathrm{CM}}$ cells and tissue-resident $\mathbf{T}_{\mathrm{EM}}$ cells}

The localization of effector memory $T\left(\mathrm{~T}_{\mathrm{EM}}\right)$ cells in peripheral tissues before infection is clearly an important factor in their ability to provide protection from a secondary infection. However, several studies have also shown that $T_{E M}$ cells and central memory $T\left(T_{C M}\right)$ cells have different effector functions in response to antigen stimulation ${ }^{51,52}$ (see the figure). Although both subsets of memory T cells produce large amounts of effector cytokines such as interferon- $\gamma$ (IFN $\gamma$ ) and tumour-necrosis factor (TNF) after antigen stimulation, a greater frequency of $\mathrm{T}_{\mathrm{CM}}$ cells also produce interleukin-2 (IL-2), which could increase their ability to proliferate in response to antigen ${ }^{94}$. By contrast, $\mathrm{T}_{\mathrm{EM}}$ cells have more potent lytic activity ex vivo compared with $\mathrm{T}_{C M}$ cells. This is probably due to the increased expression of perforin by both resting and activated $\mathrm{T}_{\mathrm{EM}}$ cells ${ }^{95}$, and because resting tissue-resident memory $T$ cells (that is, $T_{E M}$ cells) can maintain the expression of mRNA transcripts that encode cytolytic proteins ${ }^{96}$. The presence of this pre-formed mRNA enables $T_{E M}$ cells to express cytolytic proteins, such as granzyme B, more rapidly, thereby increasing their ability to rapidly kill infected cells. In addition, a small fraction of $\mathrm{T}_{E M}$ cells can express the low-affinity Fc receptor for IgG IIla (Fc $\gamma$ RIIla) (not shown), which allows them to directly mediate antibody-dependent cell-mediated cytotoxicity ${ }^{97}$.

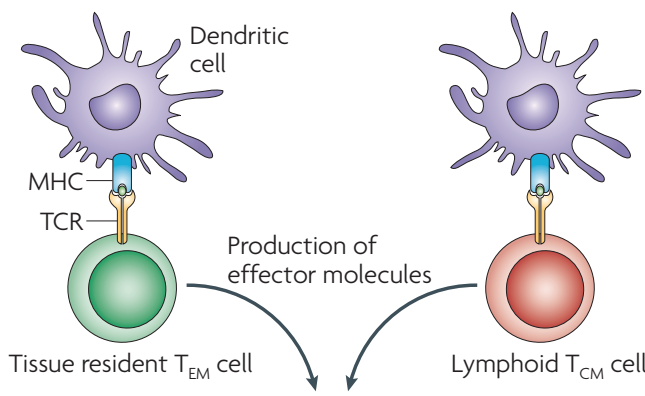

$\begin{array}{cclcc}\text { Resting } & \text { Activated } & & \text { Resting } & \text { Activated } \\ - & +++ & \text { IFN } \gamma & - & +++ \\ - & +++ & \text { TNF } & - & +++ \\ - & + & \text { IL-2 } & - & ++ \\ ++ & +++ & \text { Perforin } & - & + \\ +(\text { mRNA) } & +++ & \text { Granzyme B } & - & +\end{array}$



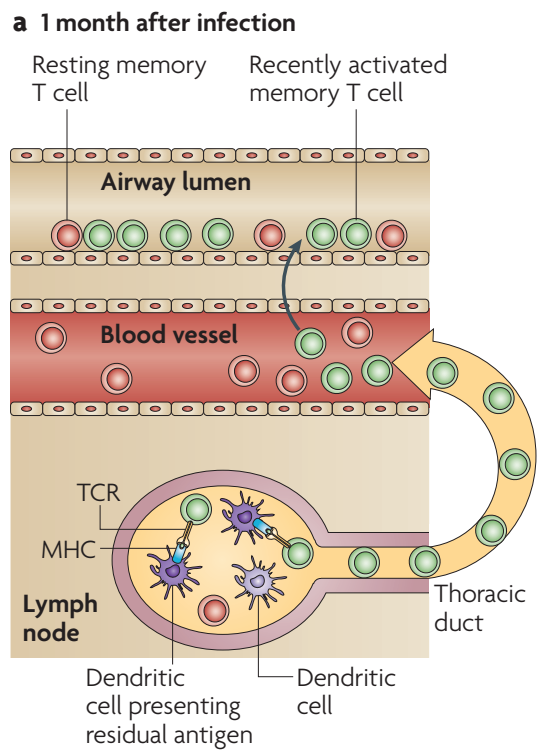
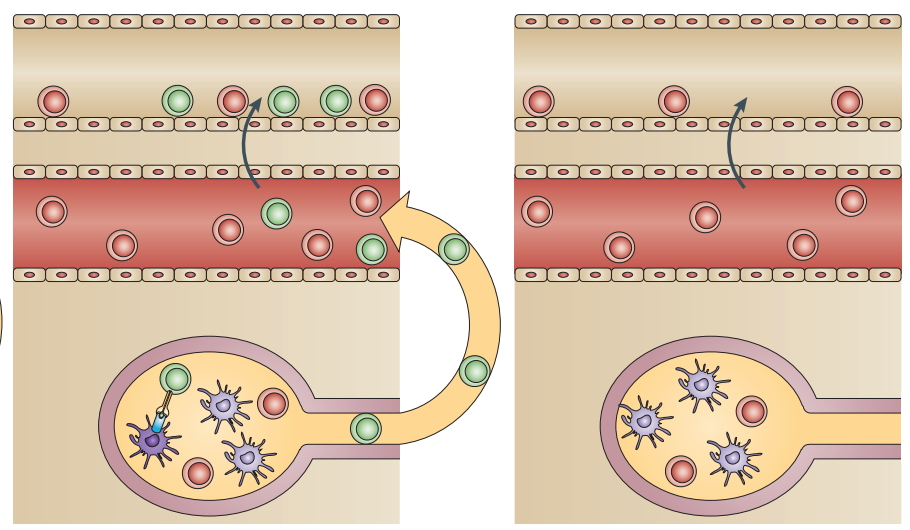

Figure 2 | The relative contribution of residual antigen to the maintenance of memory $\mathrm{CD}^{+} \mathrm{T}$ cells in the lung airways decreases over time. a | One month after the clearance of a respiratory virus infection, large numbers of antigen-specific memory $\mathrm{CD} 8^{+} \mathrm{T}$ cells are found in lung airways. Although this population contains some cells that have not recently encountered antigen (resting memory T cells), most memory $C D 8^{+} T$ cells in the airways at this time have been recently activated by residual antigen in the draining lymph node and have subsequently migrated to the airways. b | By 3 months after infection, residual antigen is mostly depleted from the draining lymph node, and the number of recently activated memory $C D 8^{+} T$ cells that contribute to the population of memory $T$ cells in the airways is subsequently decreased. $\mathbf{c | B y} 6$ months after infection, the residual antigen has been cleared from the draining lymph node and the number of antigen-specific memory $\mathrm{CD} 8^{+} \mathrm{T}$ cells in the airways has stabilized at a low level that is maintained solely by the recruitment of resting memory $T$ cells from the circulation. TCR, T-cell receptor.

destruction of the pathogen. This antigen clearance is accomplished through both elimination of the antigen source (that is, the pathogen) and the CXC-chemokine receptor 3 (CXCR3)-dependent migration of activated $\mathrm{T}$ cells to the draining lymph nodes, where they kill antigen-bearing DCs through cytolytic mechanisms ${ }^{60}$. The selective deletion of these DCs in an antigenspecific manner is thought to assist in the termination of primary immune responses, thereby limiting deleterious immunopathology. However, a recent study that investigated antigen presentation after acute influenza virus infection showed that antigen was present in the draining lymph node for several weeks after virus clearance ${ }^{61}$. These persistent antigen 'depots' contributed to the generation of influenza virus-specific $\mathrm{CD} 4^{+}$, but not $\mathrm{CD} 8^{+}$, memory $\mathrm{T}$ cells through the prolonged activation of naive $\mathrm{T}$ cells ${ }^{62}$. Importantly, similar antigen depots were also observed after the clearance of vesicular stomatitis virus infection, which indicates that persistent antigen might be a common feature of acute viral infections ${ }^{63}$. However, this same study showed that acute bacterial infection failed to result in prolonged antigen presentation, so it is currently unclear whether this phenomenon is unique to viral pathogens.

The initial report of the existence of residual antigen after influenza virus infection did not indicate that these antigen depots were involved in the generation of memory $\mathrm{CD}^{+} \mathrm{T}$ cells from naive $\mathrm{T}$ cells or if there was an effect on the antigen-specific memory $\mathrm{CD} 8^{+}$ $\mathrm{T}$-cell population that was generated during the acute infection. Additional studies have shown that memory $\mathrm{CD}^{+} \mathrm{T}$ cells in the lung airways have an activated phenotype that is indicative of recent antigen stimulation $^{64,65}$, which indicates a potential link between this peripheral population of memory $\mathrm{T}$ cells and persistent antigen presentation. Proof for this link was established using an elegant parabiotic approach that showed that the ability of blood-borne memory $\mathrm{CD}^{+} \mathrm{T}$ cells to migrate to the lung airways depended on their stimulation by antigen that persisted in the lung-draining lymph nodes ${ }^{6}$. However, this requirement for residual antigen for the recruitment of memory $\mathrm{CD}^{+} \mathrm{T}$ cells to the lung airways seemed to conflict with reports that this population was maintained in the airways in small numbers for many months after infection, long after antigen depots had been depleted. This apparent discrepancy was explained by the finding that a small number of systemic memory $\mathrm{CD}^{+} \mathrm{T}$ cells that were generated after a respiratory virus infection could migrate to the lung airways even in the absence of specific antigen ${ }^{55}$. Therefore, both antigen-dependent and antigen-independent mechanisms contribute to the maintenance of peripheral T-cell memory in the lung airways (FIG. 2). This model correlates with the observation that the number of memory $\mathrm{CD}^{+} \mathrm{T}$ cells in the lung airways gradually decreases in the first few months after infection before stabilizing at a small but sustained number ${ }^{55}$. It will be interesting to investigate whether residual antigen has a similar role in the maintenance of T-cell memory at other peripheral sites. 


\section{Protection by peripheral memory $\mathrm{T}$ cells}

Owing to their location at sites of pathogen entry, memory $\mathrm{T}$ cells that reside in peripheral tissues are thought to provide a first line of defence against secondary infections. In this capacity, antigen-specific memory $\mathrm{T}$ cells could have a dual function both as 'sentinels' at peripheral sites that alert the immune system to danger through the production of pro-inflammatory cytokines and chemokines, and to limit early pathogen replication through the destruction of infected host cells. Indeed, numerous studies have shown that memory $\mathrm{T}$ cells in the lung, skin and gut provide increased protection during a secondary infection at these sites ${ }^{67-69}$, and that the efficacy of this protection is directly linked to the number of pathogen-specific memory $\mathrm{T}$ cells in the tissue before secondary infection ${ }^{70}$.

Activation of peripheral memory T cells. Although the precise mechanisms by which memory $\mathrm{T}$ cells mediate protection against these different pathogens have not been formally shown, a common feature of memory $\mathrm{T}$ cells at peripheral sites is their ability to rapidly become activated and mediate effector functions (BOX 1). These findings have given rise to additional questions regarding the mechanisms by which memory $\mathrm{T}$ cells at peripheral sites are activated during a secondary infection, particularly as these cells are not subject to the classic method of T-cell activation by antigen-bearing DCs in the draining lymph nodes. It is possible that proinflammatory cytokines, such as IL-12, IL-18 and type I interferons, produced by innate immune cells can act directly on local memory $\mathrm{T}$ cells to induce their effector functions $\mathrm{s}^{71-73}$. In addition, non-haematopoietic cells have been shown to present antigen and induce the proliferation of effector T cells in vivo ${ }^{74}$, although there is no direct evidence that this process contributes to the activation of peripheral memory T cells. More recently, however, a seminal study has shown that resident memory T cells can be directly activated by DCs in peripheral tissues $^{75}$. Using a transplant model of herpes simplex virus reactivation, the authors observed that DCs recruited to the site of pathogen replication, together with $\mathrm{CD} 4^{+}$ $T$ cells, induced the local proliferation of antigen-specific memory CD ${ }^{+} \mathrm{T}$ cells. Together, these studies show that memory $\mathrm{T}$ cells can be activated at non-lymphoid sites by both antigen-dependent and antigen-independent mechanisms, and that each of these mechanisms probably contributes to the immune protection mediated by tissue-resident memory $\mathrm{T}$ cells.

Dynamics of peripheral $T$-cell recall responses. Once a secondary infection has been established at a peripheral site, the localized production of pro-inflammatory cytokines and chemokines recruits circulating memory $\mathrm{T}$ cells to the site of inflammation. This increased recruitment is not antigen specific, and it therefore results in the recruitment of memory $\mathrm{T}$ cells of various specificities to the site of infection ${ }^{76-78}$. However, as $\mathrm{T}$ cells specific for the invading pathogen comprise a fraction of the circulating memory T-cell pool, this process does result in a transient local increase in the number of pathogen-specific memory T cells ${ }^{79}$. Recently, the CCR5-dependent migration of circulating memory $\mathrm{T}$ cells to the lung airways during a secondary respiratory virus challenge was shown to be essential for limiting virus replication ${ }^{80}$, which shows that the rapid recruitment of memory $\mathrm{T}$ cells to the infected tissue in response to localized inflammation is important for protective immunity. In addition, transplant studies of infected ganglia have shown that recruited antigenspecific memory $\mathrm{T}$ cells can undergo extensive proliferation in peripheral tissues if antigen is present, resulting in a local amplification of pathogen-specific immune responses $^{81}$. All of these events can occur before the arrival of secondary effector $\mathrm{T}$ cells that are generated in the draining lymph nodes. Although it has not been formally shown for all peripheral tissue types, these data indicate that recall responses in peripheral tissues can be divided into distinct phases (FIG. 3). Memory T cells present in the tissue at the time of infection provide an immediate response that is further increased by memory $\mathrm{T}$ cells that are recruited from the circulation. These two phases combine to limit the early replication of the pathogen, which probably contributes to the increased survival observed during secondary infections compared with primary infections. Limiting pathogen burden before the development of a secondary effector T-cell response might also decrease immunopathology by shortening the duration of an acute infection ${ }^{82}$. So, tissue-resident memory $\mathrm{T}$ cells are not only capable of providing immediate effector function at the site of infection, but can also generate an effective secondary immune response in peripheral tissues.

\section{Implications for vaccination}

The robust protective immunity provided by memory $\mathrm{T}$ cells localized in peripheral tissues, together with the finding that memory $\mathrm{T}$ cells can be imprinted to migrate selectively to certain sites, provides hope that sitespecific vaccination strategies can be developed. This could be particularly advantageous in the case of a vaccine for $\mathrm{HIV}$, as the magnitude of the virus-specific $\mathrm{CD}^{+}$ $\mathrm{T}$-cell response in mucosal tissues has been directly linked to decreased viral load ${ }^{83,84}$. However, there have been several conflicting reports regarding the efficacy of mucosal versus systemic vaccination for the generation of peripheral T-cell memory ${ }^{85-87}$. One factor that might account for the differences observed in these and other studies is the use of protein-based versus live viral immunization strategies. Systemic vaccination with replication-competent viral vectors is considerably more efficient for generating mucosal T-cell memory than are systemic protein-based strategies, and replicationcompetent viral vectors were able to provide increased protection regardless of the route of immunization. These findings led to the question of how antigen-specific memory $\mathrm{T}$ cells could acquire tropism for tissues that did not contain replicating pathogens. An explanation was provided by a study that showed that the acquisition of tissue-homing T-cell phenotypes after different routes of live viral vaccination depended on the trafficking of activated $\mathrm{T}$ cells to antigen-free lymph nodes that were 


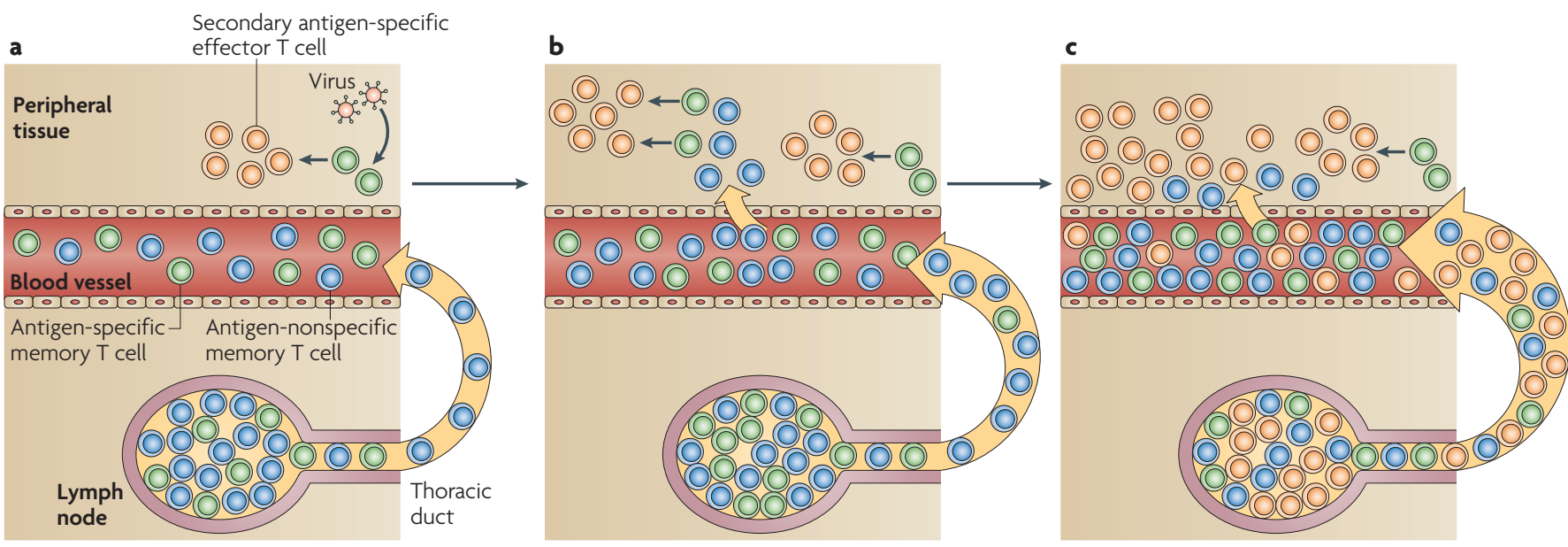

Figure 3 | Dynamics of the memory T-cell recall response in peripheral tissues. The memory T-cell response to a secondary pathogen challenge can be divided into three distinct stages. a |Antigen-specific memory T cells located in the peripheral tissue are the first to respond to an invading pathogen such as a virus. After antigen recognition, these tissue-resident memory $T$ cells can proliferate and generate a secondary effector T-cell response. $\mathbf{b} \mid$ Localized inflammation recruits both antigen-specific and antigen-nonspecific memory $T$ cells from the circulation into the infected tissue by the localized expression of chemokines. Although most of these memory T cells will not have the correct antigen specificity, and therefore are not likely to contribute to the response, the small frequency of antigen-specific memory $T$ cells that are recruited to the infected tissue can also generate a local secondary effector T-cell response after antigen encounter at this site. Also at this time (24-72 hours after infection), antigen-bearing dendritic cells have migrated to the draining lymph node, where they activate lymphoid-resident memory T cells (not shown). c | Large numbers of secondary antigen-specific effector T cells generated from lymphoid-resident memory T cells exit the draining lymph node and traffic through the bloodstream to the infected tissue. Together, these three stages limit the early replication of a pathogen during secondary infection and result in the rapid resolution of infection.

distant from the site of infection ${ }^{88}$. Once in these distant lymph nodes, the T cells not only continued to divide but also began to express homing molecules associated with the local environment. Furthermore, activated T cells that redistributed to antigen-free lymph nodes could develop into long-lived memory $\mathrm{T}$ cells ${ }^{89}$ that retained the same tissue tropism.

Although studies with live or attenuated viral immunizations have provided a wealth of information regarding correlates of vaccine-induced protection, the safety concerns surrounding these types of vaccine might preclude their widespread use in humans. So, much research is currently directed towards applying these lessons to more traditional protein-based or replicationdeficient viral vaccination strategies ${ }^{90}$. The use of new adjuvants to boost site-specific immunity is one such area of study. For example, a peptide immunogen that is linked to modified cholera toxin can elicit strong mucosal immune responses to the peptide immunogen, even when it is administered at non-mucosal sites ${ }^{91}$. In addition, the use of Toll-like receptor agonists as adjuvants can greatly increase the magnitude and quality of the immune response to prime-boost immunizations ${ }^{92}$. Finally, directed targeting of peptide-based vaccines to DCs resulted in robust T-cell memory responses that provided increased protection from subsequent pathogen challenge ${ }^{93}$. In future studies, it will be of great interest to determine whether these strategies can be modulated to take advantage of the natural mechanisms by which the immune system imparts tissue tropism to memory T cells.

\section{Concluding remarks}

A growing body of evidence indicates that memory T cells located in peripheral tissues have an important role in immune protection. These cells can mount an effective secondary T-cell response at the site of infection, thereby eliminating the time required for antigen-presenting cells to traffic to the draining lymph node before eliciting a secondary effector T-cell response. The ability to mount these immediate responses leads to increased pathogen clearance, and the importance of peripheral memory $\mathrm{T}$ cells in this process is evident from the finding that the loss of pathogen-specific memory $\mathrm{T}$ cells in the periphery over time is accompanied by decreased protective immunity. There is still much we do not understand regarding the dynamics and mechanisms of protection mediated by peripheral memory $\mathrm{T}$ cells, but the advent of new intravital techniques could provide new insights by allowing us to visualize these responses directly and in greater detail.

Although the distinction between lymphoid and peripheral memory $\mathrm{T}$ cells is often imprecise, it is clear that individual tissues can govern the tropism of pathogen-specific T cells that arise after localized infections. This is true for the gut and skin, and the search for tissue-specific T-cell phenotypes at other peripheral sites is currently an area of intense investigation. The potential benefit of understanding the factors that influence memory T-cell migration to specific sites is considerable, as this information could be used to increase vaccine efficacy against pathogens that selectively infect certain tissues, and it could also provide new therapeutic avenues for the amelioration of site-specific autoimmunity. 
1. Masopust, D. et al. Activated primary and memory CD8 T cells migrate to nonlymphoid tissues regardless of site of activation or tissue of origin. J. Immunol. 172, 4875-4882 (2004)

2. Jamieson, T. et al. The chemokine receptor D6 limits the inflammatory response in vivo. Nature Immunol. 6, 403-411 (2005)

3. Ariel, A. et al. Apoptotic neutrophils and T cells sequester chemokines during immune response resolution through modulation of CCR5 expression. Nature Immunol. 7, 1209-1216 (2006).

4. Badovinac, V. P., Porter, B. B. \& Harty, J. T. Programmed contraction of $\mathrm{CD}^{+} \mathrm{T}$ cells after infection. Nature Immunol. 3, 619-626 (2002).

5. Kaech, S. M. et al. Selective expression of the interleukin 7 receptor identifies effector CD8 T cells that give rise to long-lived memory cells. Nature Immunol. 4, 1191-1198 (2003)

6. Flynn, K. J. et al. Virus-specific CD8 ${ }^{+} \mathrm{T}$ cells in primary and secondary influenza pneumonia. Immunity 8, 683-691 (1998).

7. Hogan, R. J. et al. Activated antigen-specific $\mathrm{CD}^{+}$ $\mathrm{T}$ cells persist in the lungs following recovery from respiratory virus infections. J. Immunol. 166 1813-1822 (2001).

8. Reinhardt, R. L., Khoruts, A., Merica, R., Zell, T. $\delta$ Jenkins, M. K. Visualizing the generation of memory CD4 T cells in the whole body. Nature 410, 101-105 (2001).

9. Koelle, D. M. et al. Expression of cutaneous lymphocyte-associated antigen by $\mathrm{CD} 8^{+} \mathrm{T}$ cells specific for a skin-tropic virus. J. Clin. Invest. 110 537-548 (2002)

10. Mackay, C. R. et al. Tissue-specific migration pathways by phenotypically distinct subpopulations of memory T cells. Eur. J. Immunol. 22, 887-895 (1992).

11. Williams, M. B. \& Butcher, E. C. Homing of naive and memory T lymphocyte subsets to Peyer's patches, lymph nodes, and spleen. J. Immunol. 159 1746-1752 (1997).

12. Klonowski, K. D. et al. Dynamics of blood-borne CD8 memory T cell migration in vivo. Immunity 20 , 551-562 (2004)

This study used a parabiotic approach to show that the distribution of memory $\mathrm{T}$ cells in peripheral tissues is not equal, providing convincing evidence that there is restricted entry of circulating memory T cells into certain sites.

13. Sanders, M. E. et al. Human memory T lymphocytes express increased levels of three cell adhesion molecules (LFA-3, CD2, and LFA-1) and three other molecules (UCHL1, CDw29, and Pgp-1) and have enhanced IFN- $\gamma$ production. J. Immunol. 140, 1401-1407 (1988).

14. Shimizu, Y., Van Seventer, G. A., Horgan, K. J. \& Shaw, S. Regulated expression and binding of three VLA ( $\beta 1)$ integrin receptors on T cells. Nature 345 , 250-253 (1990)

15. Hirata, T. et al. P-selectin glycoprotein ligand 1 (PSGL-1) is a physiological ligand for E-selectin in mediating T helper 1 lymphocyte migration. J. Exp. Med. 192, 1669-1676 (2000).

16. Bonder, C. S., Clark, S. R., Norman, M. U., Johnson, P. $\&$ Kubes, P. Use of CD 44 by CD4+ Th 1 and Th2 lymphocytes to roll and adhere. Blood 107 4798-4806 (2006).

17. Ostermann, G., Weber, K. S., Zernecke, A., Schroder, A $\&$ Weber, C. JAM-1 is a ligand of the $\beta_{2}$ integrin LFAinvolved in transendothelial migration of leukocytes. Nature Immunol. 3, 151-158 (2002)

18. Abitorabi, M. A. et al. Differential expression of homing molecules on recirculating lymphocytes from sheep gut, peripheral, and lung lymph. J. Immunol. 156, 3111-3117 (1996).

19. Marth, J. D. \& Grewal, P. K. Mammalian glycosylation in immunity. Nature Rev. Immunol. 8, 874-887 (2008).

20. Rodrigo Mora, J. \& Von Andrian, U. H. Specificity and plasticity of memory lymphocyte migration. Curr. Top. Microbiol. Immunol. 308, 83-116 (2006).

21. Sigmundsdottir, H. \& Butcher, E. C. Environmental cues, dendritic cells and the programming of tissueselective lymphocyte trafficking. Nature Immunol. 9, 981-987 (2008)

22. Fuhlbrigge, R. C., Kieffer, J. D., Armerding, D. \& Kupper, T. S. Cutaneous lymphocyte antigen is a specialized form of PSGL-1 expressed on skin-homing T cells. Nature 389, 978-981 (1997)

23. Picker, L. J et al. Differential expression of homingassociated adhesion molecules by $\mathrm{T}$ cell subsets in man. J. Immunol. 145, 3247-3255 (1990).
24. Campbell, J. J. et al. The chemokine receptor CCR4 in vascular recognition by cutaneous but not intestinal memory T cells. Nature 400, 776-780 (1999).

25. Reiss, Y., Proudfoot, A. E., Power, C. A., Campbell, J. J. $\&$ Butcher, E. C. CC chemokine receptor (CCR) 4 and the CCR 10 ligand cutaneous T cell-attracting chemokine (CTACK) in lymphocyte trafficking to inflamed skin. J. Exp. Med. 194, 1541-1547 (2001).

26. Berlin, C. et al. a $4 \beta 7$ integrin mediates lymphocyte binding to the mucosal vascular addressin MAdCAM-1. Cell 74, 185-195 (1993).

27. Lefrancois, L. et al. The role of $\beta 7$ integrins in CD8 $\mathrm{T}$ cell trafficking during an antiviral immune response. J. Exp. Med. 189, 1631-1638 (1999).

28. Zabel, B. A. et al. Human G protein-coupled receptor GPR-9-6/CC chemokine receptor 9 is selectively expressed on intestinal homing T lymphocytes, mucosal lymphocytes, and thymocytes and is required for thymusexpressed chemokine-mediated chemotaxis. J. Exp. Med. 190, 1241-1256 (1999)

29. Kunkel, E. J. et al. Lymphocyte CC chemokine receptor 9 and epithelial thymus-expressed chemokine (TECK) expression distinguish the small intestinal immune compartment: epithelial expression of tissuespecific chemokines as an organizing principle in regional immunity. J. Exp. Med. 192, 761-768 (2000)

30. Iwata, M. et al. Retinoic acid imprints gut-homing specificity on T cells. Immunity 21, 527-538 (2004)

31. Mora, J. R. et al. Reciprocal and dynamic control of CD8 T cell homing by dendritic cells from skin- and gut-associated lymphoid tissues. J. Exp. Med. 201 303-316 (2005)

32. Dudda, J. C. et al. Dendritic cells govern induction and reprogramming of polarized tissue-selective homing receptor patterns of T cells: important roles for soluble factors and tissue microenvironments. Eur. J. Immunol. 35, 1056-1065 (2005).

33. Jiang, J. Q., He, X. S., Feng, N. \& Greenberg, H. B. Qualitative and quantitative characteristics of rotavirus-specific CD8 T cells vary depending on the route of infection. J. Virol. 82, 6812-6819 (2008).

34. Campbell, D. J. \& Butcher, E. C. Rapid acquisition of tissue-specific homing phenotypes by $\mathrm{CD} 4^{+} \mathrm{T}$ cells activated in cutaneous or mucosal lymphoid tissues. J. Exp. Med. 195, 135-141 (2002)

35. Mora, J. R. et al. Selective imprinting of gut-homing T cells by Peyer's patch dendritic cells. Nature 424 88-93 (2003).

36. Edele, F. et al. Cutting edge: instructive role of peripheral tissue cells in the imprinting of T cell homing receptor patterns. J. Immunol. 181 3745-3749 (2008).

37. Ahrendt, M., Hammerschmidt, S. I., Pabst, O., Pabst, R. $\&$ Bode, U. Stromal cells confer lymph node-specific properties by shaping a unique microenvironment influencing local immune responses. J. Immunol. 181, 1898-1907 (2008)

38. Hammerschmidt, S. I. et al. Stromal mesenteric lymph node cells are essential for the generation of guthoming T cells in vivo. J. Exp. Med. 205, 2483-2490 (2008)

By transplanting distant lymph nodes into the mesenteries, this study clearly showed that the stromal cells in regional lymph nodes mediate the control of tissue-specific imprinting.

39. Calzascia, T. et al. Homing phenotypes of tumor-specific CD8 T cells are predetermined at the tumor site by crosspresenting APCs. Immunity 22, 175-184 (2005).

40. Kivisakk, P. et al. Human cerebrospinal fluid central memory $\mathrm{CD}_{4}{ }^{+} \mathrm{T}$ cells: evidence for trafficking through choroid plexus and meninges via P-selectin. Proc. Nat Acad. Sci. USA 100, 8389-8394 (2003).

41. van der Most, R. G., Murali-Krishna, K. \& Ahmed, R. Prolonged presence of effector-memory CD8 T cells in the central nervous system after dengue virus encephalitis. Int. Immunol. 15, 119-125 (2003).

42. de Bree, G. J. et al. Selective accumulation of differentiated $\mathrm{CD} 8{ }^{+} \mathrm{T}$ cells specific for respiratory viruses in the human lung. J. Exp. Med. 202, 1433-1442 (2005)

This study provided the first convincing evidence that human memory $\mathrm{T}$ cells specific for respiratory pathogens are selectively localized to the lungs, which indicates that memory $T$ cells can be imprinted to migrate to the respiratory tract.

43. Ray, S. J. et al. The collagen binding $\alpha 1 \beta 1$ integrin VLA-1 regulates CD8 T cell-mediated immune protection against heterologous influenza infection. Immunity 20, 167-179 (2004).
44. Surh, C. D., Boyman, O., Purton, J. F. \& Sprent, J. Homeostasis of memory T cells. Immunol. Rev. 211 154-163 (2006)

45. Hogan, R. J. et al. Long-term maintenance of virusspecific effector memory $C D 8^{+} \mathrm{T}$ cells in the lung airways depends on proliferation. J. Immunol. 169 , 4976-4981 (2002).

46. Harris, N. L., Watt, V., Ronchese, F. \& Le Gros, G. Differential $\mathrm{T}$ cell function and fate in lymph node and nonlymphoid tissues. J. Exp. Med. 195, 317-326 (2002).

47. Wright, J. R. Immunoregulatory functions of surfactant proteins. Nature Rev. Immunol. 5, 58-68 (2005).

48. Shen, C. H. et al. Loss of IL-7R and IL-15R expression is associated with disappearance of memory $T$ cells in respiratory tract following influenza infection. J. Immunol. 180, 171-178 (2008)

49. Wang, X. Z. et al. Virus-specific CD8 T cells in peripheral tissues are more resistant to apoptosis than those in lymphoid organs. Immunity 18 , 631-642 (2003)

This paper provided an explanation for the persistence of memory T cells in the harsh conditions of non-lymphoid sites by showing that memory $\mathrm{T}$ cells in the periphery are more resistant to apoptosis than those in lymphoid tissues.

50. Kassiotis, G. \& Stockinger, B. Anatomical heterogeneity of memory $\mathrm{CD} 4{ }^{+} \mathrm{T}$ cells due to reversible adaptation to the microenvironment. J. Immunol. 173, 7292-7298 (2004).

51. Sallusto, F., Lenig, D., Forster, R., Lipp, M. \& Lanzavecchia, A. Two subsets of memory $T$ lymphocytes with distinct homing potentials and effector functions. Nature 401, 708-712 (1999).

52. Masopust, D., Vezys, V., Marzo, A. L. \& Lefrancois, L. Preferential localization of effector memory cells in nonlymphoid tissue. Science 291, 2413-2417 (2001).

53. Marzo, A. L. et al. Initial T cell frequency dictates memory CD8+ $\mathrm{T}$ cell lineage commitment. Nature Immunol. 6, 793-799 (2005)

54. Vezys, V. et al. Memory CD8 T-cell compartment grows in size with immunological experience. Nature 457 196-199 (2008)

55. Kohlmeier, J. E., Miller, S. C. \& Woodland, D. L. Cutting edge: Antigen is not required for the activation and maintenance of virus-specific memory CD8 $\mathrm{T}$ cells in the lung airways. J. Immunol. 178 4721-4725 (2007)

56. Masopust, D., Vezys, V., Wherry, E. J., Barber, D. L. ¿ Ahmed, R. Cutting edge: Gut microenvironment promotes differentiation of a unique memory CD8 T cell population. J. Immunol. 176, 2079-2083 (2006).

57. Ely, K. H., Cookenham, T., Roberts, A. D. \& Woodland, D. L. Memory T cell populations in the lung airways are maintained by continual recruitment. J. Immunol. 176, 537-543 (2006)

58. Richards, H., Longhi, M. P., Wright, K., Gallimore, A. \& Ager, A. CD62L (L-selectin) down-regulation does not affect memory $T$ cell distribution but failure to shed compromises anti-viral immunity. J. Immunol. 180, 198-206 (2008)

59. Marzo, A. L., Yagita, H. \& Lefrancois, L. Cutting edge: Migration to nonlymphoid tissues results in functional conversion of central to effector memory CD8 T cells. J. Immunol. 179, 36-40 (2007).

60. Guarda, G. et al. L-selectin-negative CCR7- effector and memory $\mathrm{CD} 8{ }^{+} \mathrm{T}$ cells enter reactive lymph nodes and kill dendritic cells. Nature Immunol. 8, 743-752 (2007).

61. Jelley-Gibbs, D. M. et al. Unexpected prolonged presentation of influenza antigens promotes CD4 T cell memory generation. J. Exp. Med. 202, 697-706 (2005)

62. Jelley-Gibbs, D. M. et al. Persistent depots of influenza antigen fail to induce a cytotoxic CD8 T cell response. J. Immunol. 178, 7563-7570 (2007).

63. Turner, D. L., Cauley, L. S., Khanna, K. M. \& Lefrancois, L. Persistent antigen presentation after acute vesicular stomatitis virus infection. J. Virol. $\mathbf{8 1}$ 2039-2046 (2007).

64. Marshall, D. R. et al. Measuring the diaspora for virusspecific CD8 ${ }^{+}$T cells. Proc. Natl Acad. Sci. USA 98 6313-6318 (2001)

65. Hogan, R. J. et al. Activated antigen-specific CD8 $T$ cells persist in the lungs following recovery from respiratory virus infections. J. Immunol. 166 , 1813-1822 (2001). 
66. Zammit, D. J., Turner, D. L., Klonowski, K. D., Lefrancois, L. \& Cauley, L. S. Residual antigen presentation after influenza virus infection affects CD8 $\mathrm{T}$ cell activation and migration. Immunity $\mathbf{2 4}$, 439-449 (2006)

This study extended on the previous finding that antigen was presented long after influenza virus clearance to show that residual antigen in the draining lymph nodes is important for the maintenance of memory $T$ cells in the lung airways.

67. Hogan, R. J. et al. Protection from respiratory virus infections can be mediated by antigen-specific CD4 $\mathrm{T}$ cells that persist in the lungs. J. Exp. Med. 193 981-986 (2001)

68 Stittelaar K. J et al. Modified vaccinia virus Ankara protects macaques against respiratory challenge with monkeypox virus. J. Virol. 79, 7845-7851 (2005)

69. Xu, R., Johnson, A. J., Liggitt, D. \& Bevan, M. J. Cellular and humoral immunity against vaccinia virus infection of mice. J. Immunol. 172, 6265-6271 (2004).

70. Liang, S., Mozdzanowska, K., Palladino, G. $\&$ Gerhard, W. Heterosubtypic immunity to influenza type A virus in mice. Effector mechanisms and their longevity. J. Immunol. 152, 1653-1661 (1994). This classic study showed that the number of memory $\mathrm{T}$ cells maintained in the periphery dictates the level of protection provided during a secondary pathogen challenge, and that the decreasing number of these cells over time has a detrimental effect on T-cell-mediated protection.

71. Raue, H. P., Brien, J. D., Hammarlund, E. \& Slifka, M. K. Activation of virus-specific CD8 ${ }^{+} \mathrm{T}$ cells by lipopolysaccharide-induced IL-12 and IL-18. J. Immunol. 173, 6873-6881 (2004).

72. Kamath, A. T., Sheasby, C. E. \& Tough, D. F. Dendritic cells and NK cells stimulate bystander T cell activation in response to TLR agonists through secretion of IFN $\alpha \beta$ and IFN- $\gamma$. J. Immunol. 174, 767-776 (2005).

73 Denton, A. E., Doherty, P. C., Turner, S. J. \& La Gruta, N. L. IL-18, but not IL-12, is required for optimal cytokine production by influenza virus-specific CD8+ T cells. Eur. J. Immunol. 37, 368-375 (2007).

74. Thomas, S., Kolumam, G. A. \& Murali-Krishna, K. Antigen presentation by nonhemopoietic cells amplifies clonal expansion of effector CD8 T cells in a pathogen-specific manner. J. Immunol. 178, 5802-5811 (2007)

75. Wakim, L. M., Waithman, J., van Rooijen, N., Heath, W. R. \& Carbone, F. R. Dendritic cell-induced memory $\mathrm{T}$ cell activation in nonlymphoid tissues. Science $\mathbf{3 1 9}$, 198-202 (2008)

Using an elegant transplantation model, this study provided the first evidence that memory $\mathrm{T}$ cells located in peripheral tissues can generate a localized secondary effector T-cell response without first trafficking to lymphoid tissues.

76. Topham, D. J., Castrucci, M. R., Wingo, F. S., Belz, G. T. $\&$ Doherty, P. C. The role of antigen in the localization of naive, acutely activated, and memory $\mathrm{CD} 8+\mathrm{T}$ cells to the lung during influenza pneumonia. J. Immunol. 167, 6983-6990 (2001)
77. Ely, K. H. et al. Nonspecific recruitment of memory $\mathrm{CD} 8+\mathrm{T}$ cells to the lung airways during respiratory virus infections. J. Immunol. 170, 1423-1429 (2003)

78. Chen, A. M., Khanna, N., Stohlman, S. A. \& Bergmann, C. C. Virus-specific and bystander CD8 T cells recruited during virus-induced encephalomyelitis. J. Virol. 79, 4700-4708 (2005)

79. Heidema, J. et al. Dynamics of human respiratory virus-specific CD8 ${ }^{+} \mathrm{T}$ cell responses in blood and airways during episodes of common cold. J. Immunol. 181, 5551-5559 (2008)

80. Kohlmeier, J. E. et al. The chemokine receptor CCR5 plays a key role in the early memory $\mathrm{CD}^{+} \mathrm{T}$ cell response to respiratory virus infections. Immunity 29 101-113 (2008).

By identifying and then inhibiting the mechanism of memory T-cell recruitment to the lung airways, this study showed the importance of memory T-cell recruitment for the early control of pathogen replication during a secondary infection.

81. Wakim, L. M., Gebhardt, T., Heath, W. R. \& Carbone, F. R. Cutting edge: Local recall responses by memory $T$ cells newly recruited to peripheral nonlymphoid tissues. J. Immunol. 181, 5837-5841 (2008).

82. Chen, H. D. et al. Memory CD8 ${ }^{+} \mathrm{T}$ cells in heterologous antiviral immunity and immunopathology in the lung. Nature Immunol. 2, 1067-1076 (2001)

83. Kaul, R. et al. HIV-1-specific mucosal CD8 ${ }^{+}$ lymphocyte responses in the cervix of HIV-1-resistan prostitutes in Nairobi. J. Immunol. 164, 1602-161 (2000).

84. Belyakov, I. M. et al. Mucosal AIDS vaccine reduces disease and viral load in gut reservoir and blood after mucosal infection of macaques. Nature Med. 7, 1320-1326 (2001)

85. Gallichan, W. S. \& Rosenthal, K. L. Long-lived cytotoxic T lymphocyte memory in mucosal tissues after mucosal but not systemic immunization. J. Exp. Med. 184, 1879-1890 (1996).

86. Stevceva, L. et al. Both mucosal and systemic routes of immunization with the live, attenuated NYVAC/ simian immunodeficiency virus SIV(gpe) recombinant vaccine result in gag-specific CD8 ${ }^{+}$T-cell responses in mucosal tissues of macaques. J. Virol. 76 11659-11676 (2002)

87. Abel, K. et al. Simian-human immunodeficiency virus SHIV89.6-induced protection against intravaginal challenge with pathogenic SIVmac239 is independent of the route of immunization and is associated with a combination of cytotoxic T-lymphocyte and alpha interferon responses. J. Virol. 77, 3099-3118 (2003)

88. Liu, L., Fuhlbrigge, R. C., Karibian, K., Tian, T. \& Kupper, T. S. Dynamic programming of CD8 ${ }^{+} \mathrm{T}$ cell trafficking after live viral immunization. Immunity 25 511-520 (2006).

Using different vaccination routes, this paper showed that antigen-specific $T$ cells that had disseminated from the priming lymph nodes into distant lymph nodes were able to acquire new homing phenotypes based on the local environment.

89. Brinkman, C. C., Sheasley-O'Neill, S. L., Ferguson, A. R. \& Engelhard, V. H. Activated CD8 T cells redistribute to antigen-free lymph nodes and exhibit effector and memory characteristics. J. Immunol. 181 1814-1824 (2008).

90. Seder, R. A., Darrah, P. A. \& Roederer, M. T-cell quality in memory and protection: implications for vaccine design. Nature Rev. Immunol. 8, 247-258 (2008).

91. Luci, C. et al. Dendritic cell-mediated induction of mucosal cytotoxic responses following intravaginal immunization with the nontoxic B subunit of cholera toxin. J. Immunol. 176, 2749-2757 (2006).

92. Wille-Reece, U. et al. Toll-like receptor agonists influence the magnitude and quality of memory $T$ cell responses after prime-boost immunization in nonhuman primates. J. Exp. Med. 203, 1249-1258 (2006).

93. Trumpfheller, C. et al. Intensified and protective CD4 ${ }^{+}$ T cell immunity in mice with anti-dendritic cell HIV gag fusion antibody vaccine. J. Exp. Med. 203, 607-617 (2006).

94. MacLeod, M. K. et al. CD4 memory T cells divide poorly in response to antigen because of their cytokine profile. Proc. Natl Acad. Sci. USA 105 14521-14526 (2008).

95. Champagne, P. et al. Skewed maturation of memory HIV-specific CD8 T lymphocytes. Nature $\mathbf{4 1 0}$ 106-111 (2001)

96. Mintern, J. D., Guillonneau, C., Carbone, F. R., Doherty, P. C. \& Turner, S. J. Cutting edge: Tissueresident memory CTL down-regulate cytolytic molecule expression following virus clearance. J. Immunol. 179, 7220-7224 (2007).

97. Clemenceau, B. et al. Effector memory $\alpha \beta \mathrm{T}$ lymphocytes can express Fc $\gamma$ RIIIa and mediate antibody-dependent cellular cytotoxicity. J. Immunol. $180,5327-5334$ (2008)

\section{Acknowledgements}

We thank M. Blackman for critical review of the manuscrip and A. Roberts for graphic design of original figures. This work was supported by funds from the National Institutes of Health, USA, and the Trudeau Institute, USA.

\section{DATABASES}

Entrez Gene: http://www.ncbi.nlm.nih.gov/entrez/query. fcgi?db=gene

a 437-integrin $\mid$ CCL17 $\mid$ CCL25 $\mid$ CCL27 |CCR4 |CCR5 CCR7 |CCR9 | CCR10 | CD44 |CD62L ICLA|CXCR3 | IL-7 IL-15 | LFA1 | MADCAM1 | PSGL1 |SFTPA1 |SFTPD |VLA1 |

FURTHER INFORMATION

David Woodland's homepage: http://trudeauinstitute.org/ dynamicPages/faculty.cfm?action=details\&ID=106\&navTab e=tier2nav\&facultylD $=14$

ALL LINKS ARE ACTIVE IN THE ONLINE PDF 